\title{
Colorectal Carcinogenesis and Animal Models
}

\section{Shubhankar Suman and Kamal Datta*}

Department of Biochemistry and Molecular \& Cell Biology, Georgetown University, Research Building, Room E518, 3970 Reservoir Rd., NW, Washington, DC 200571468, USA

\section{Editorial}

The incidence of colorectal cancer (CRC), common in Western developed countries, is rapidly rising in countries with recent economic growth. CRC remains a common cancer both in men and in women around the world, and among all the cancers its incidence in men is fourth and in women third. In the US alone, it is predicted that 143,460 people will be diagnosed with CRC in 2012 [1] with the frequency of precancerous lesions being present in about $10 \%$ of adults at age 40. The incidence of precancerous lesions increases to $\sim 25 \%$ in older adults, and in 2007 about 53,219 people died of CRC in the US [2]. It is important to recognize that CRC is still the second leading cause of cancer related deaths and was responsible for $10 \%$ of the mortality worldwide [3]. The lifetime risk of developing colon cancer in United States is about 1 in 20 (5.1\%) [1] and factors known to increase the risk of developing CRC include: age, family history, smoking, dietary habits, physical activity, inflammatory bowel syndrome, alcohol and virus infection.

Colorectal carcinogenesis occurs as result of complex genetic and epigenetic alterations, leading to uncontrolled proliferation of crypts, which have very high cellular turnover occurring under tight regulation of signaling events. Depending solely on extrapolation from cell culture models to define the multifaceted carcinogenic precursor events of CRC carries much uncertainty. Cell culture models, although useful in understanding basic molecular events of CRC, lack threedimensional tissue architecture and associated microenvironment. Animal models relevant to the molecular pathways involved in human CRC have played important roles in our understanding of colorectal carcinogenesis and allowed testing of factors affecting the frequency of the disease in human populations. Animal models have also greatly facilitated the development of newer chemopreventive and therapeutic strategies to reduce mortality and incidence related to CRC.

Supported by genome level changes which confer growth advantage and clonal expansion, CRC is thought to progress from aberrant crypt foci to adenocarcinoma over long periods of time. To date, much of the signaling pathway alterations in CRC have been delineated and the most commonly altered is the Wnt/APC/ $\beta$-catenin/TCF-4 (T-cell factor-4) pathway, where APC (adenomatous polyposis coli) mutation is the most prevalent $[4,5]$. With dysfunctional APC protein, $\beta$-catenin translocates into the nucleus, complex with TCF-4, and inappropriately activates the transcription of genes leading to uncontrolled proliferation (Figure 1). Although most CRC display APC mutation, stabilization mutations in $\beta$-catenin that block its degradation has also been observed, and others may have mutations in genes with function analogous to APC such as AXIN1, AXIN2, TCF7L2 or the NKD1 [6]. Apart from the defects in the Wnt/APC/ $\beta$-catenin/TCF4 signaling pathway, other pathways commonly altered in CRC are TGF- $\beta$ and DCC (deleted in colorectal cancer). Furthermore, in CRC, genes like K-RAS, RAF and phosphatidylinositol-3-Kinase (PI3K), have also been mutated resulting in increased cellular proliferation $[4,6]$. Mutation in tumor suppressor PTEN, which normally inhibits PI3K has also been reported to contribute to colorectal carcinogenesis. On the contrary, mutations in tumor suppressor p53, observed in 40-50\% of CRC [7], are known to be a late event in colorectal carcinogenesis and possibly develop during adenoma-carcinoma transition. Most widely studied germline mutations in human are familial adenomatous polyp (FAP) and Lynch syndrome, which cause increased risk of CRC. Recently, MYH-associated polyposis (MAP) and MSH6 mutation leading to an atypical Lynch syndrome is reported to carry increase risk of CRC $[8,9]$. A number of animal models have been developed representing these signaling events in CRC and these models have played crucial roles towards our understanding of CRC.

Understanding of the molecular pathogenesis of colorectal carcinogenesis was significantly expedited by using animal models. Initial animal models of CRC involved chemical-induced models, which have found wide use in testing carcinogens. Subsequent development of the transgenic mouse model helped, not only in recognizing

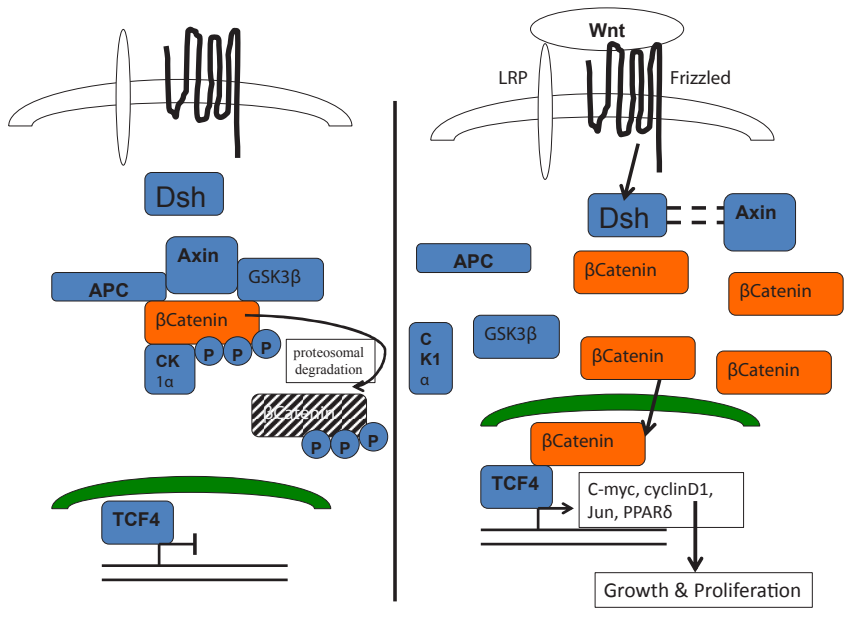

Figure 1: Schematic representation of Wnt/APC/ $\beta$-catenin/TCF-4 pathway. The left panel shows functional APC in complex with axin and GSK3 $\beta$ regulating $\beta$-catenin activity and the right panel shows non-functional APC allowing translocation of $\beta$-catenin to nucleus where in cooperation with TCF-4 it causes uncontrolled proliferation.

*Corresponding author: Kamal Datta, Department of Biochemistry and Molecular \& Cellular Biology, Georgetown University, Research Building, Room E518, 3970 Reservoir Rd., NW, Washington, DC 20057-1468, USA; Tel: 202-687-7956; E-mail: kd257@georgetown.edu

Received March 22, 2012; Accepted March 26, 2012; Published March 28, 2012

Citation: Suman S, Datta K (2012) Colorectal Carcinogenesis and Animal Models. J Carcinogene Mutagene 3:e104. doi:10.4172/2157-2518.1000e104

Copyright: ( 2012 Suman S, et al. This is an open-access article distributed under the terms of the Creative Commons Attribution License, which permits unrestricted use, distribution, and reproduction in any medium, provided the original author and source are credited. 
CRC initiation and progression events, but also in testing candidate chemopreventive agents. Mutant models and xeno-transplant models were instrumental in defining the tumor progression modifiers and tumor cell interactions. In the last few decades, many murine models have been established that are useful for the investigation of initiation, expansion and progression of CRC. In most of these models, Wnt signaling, mismatch repair, and TGF $\beta$ pathways are targeted, not only to understand initiation and progression of CRC, but also to evaluate various pharmaceutical and biological agents for prevention and treatment of CRC. Common carcinogens like AOM (azoxymethane), DMH (dimethylhydrazine), or PhIP (2-amino-1-methyl-6phenylimidazo $[4,5-b]$ pyridine) were used to develop rat models of CRC $[10,11]$. The $\mathrm{APC}^{\mathrm{Min} /+}$ (Min: multiple intestinal neoplasia) is the first heritable mouse model of CRC, developed in 1990 as a result of ethylnitrosourea (ENU)-induced germline truncating mutation at the codon 850 of the APC gene [12,13]. In the C57BL/6J mouse background $\mathrm{APC}^{\mathrm{Min} /+}$ mice develop about 30 small intestinal polyps with occasional adenocarcinoma and essentially no tumor in the colon. Although this contrasts human FAP, where most of the adenomas are in the colon and these adenomas certainly progress to invasive adenocarcinoma, the $\mathrm{APC}^{\mathrm{Min} /+}$ models due to their phenotypic and histopathological similarities to human intestinal neoplasm are used not only to test therapeutic and chemopreventive interventions but also to understand the role of APC gene in CRC [14]. The $\mathrm{APC}^{\mathrm{Min} /+}$ models also proved to be important for the study of genetic modifiers-of-Min (Mom) locus. The hereditary non-polyposis colorectal cancer (HNPCC), an inherited condition with inactivated DNA mismatch repair (MMR) genes, like MLH1, MSH2, MSH6, and PMS2 [15,16], leads to the development of a variety of cancers including that of the colon [17]. A number of mouse models with loss of function of MMR genes have been generated and mice lacking Mlh1, Msh2 and Msh6 develop tumors in the stomach, small intestine, and colon. The TGF- $\beta$ signaling, involved in cellular differentiation, growth suppression, deposition of extracellular matrix and apoptosis, is known to play important roles in colorectal carcinogenesis. Mouse models related to the TGF- $\beta$ pathway have been used to delineate the multifaceted role of this pathway during colorectal carcinogenesis. CRC xenograft models are generated by inserting cells subcutaneously, intrasplenically, or into the renal capsule and are commonly used to understand the pathogenesis of the human disease. The orthotopic mouse model, where colorectal cancer cells or tumor tissue is placed into the intestinal sub-mucosa [18], mimics metastatic behavior and microenvironments of human CRC.

Animal models offer distinct advantages in studying numerous adenomas under an identical genetic background and defined experimental condition. In the future a combination of dietary and genetic manipulation of the small experimental animals is called for to characterize various factors that contribute to colorectal carcinogenesis in humans.

\section{References}

1. http://www.cancer.org/

2. http://www.cdc.gov/cancer/colorectal/statistics.

3. Einspahr JG, Alberts DS, Gapstur SM, Bostick RM, Emerson SS, et al. (1997) Surrogate end-point biomarkers as measures of colon cancer risk and their use in cancer chemoprevention trials. Cancer Epidemiol Biomarkers Prev 6: 37-48.

4. Kinzler KW, Vogelstein B (1996) Lessons from hereditary colorectal cancer. Cell 87: 159-170.
5. Peignon G, Durand A, Cacheux W, Ayrault O, Terris B et al. (2011) Complex interplay between beta-catenin signalling and Notch effectors in intestinal tumorigenesis. Gut 60: 166-176.

6. Grady WM, Carethers JM (2008) Genomic and epigenetic instability in colorectal cancer pathogenesis. Gastroenterology 135: 1079-1099.

7. Liu Y, Bodmer WF (2006) Analysis of P53 mutations and their expression in 56 colorectal cancer cell lines. Proc Natl Acad Sci USA 103: 976-981.

8. Goodenberger M, Lindor NM (2011) Lynch syndrome and MYH-associated polyposis: review and testing strategy. J Clin Gastroenterol 45: 488-500.

9. Kastrinos F, Syngal S (2007) Recently identified colon cancer predispositions: MYH and MSH6 mutations. Semin Oncol 34: 418-424.

10. Corpet DE, Pierre F (2003) Point: From animal models to prevention of colon cancer. Systematic review of chemoprevention in min mice and choice of the model system. Cancer Epidemiol Biomarkers Prev 12: 391-400.

11. Corpet DE, Pierre F (2005) How good are rodent models of carcinogenesis in predicting efficacy in humans? A systematic review and meta-analysis of colon chemoprevention in rats, mice and men. Eur J Cancer 41: 1911-1922.

12. Moser AR, Pitot HC, Dove WF (1990) A dominant mutation that predisposes to multiple intestinal neoplasia in the mouse. Science 247: 322-324.

13. Moser AR, Dove WF, Roth KA, Gordon JI (1992) The Min (multiple intestina neoplasia) mutation: its effect on gut epithelial cell differentiation and interaction with a modifier system. J Cell Biol 116: 1517-1526.

14. Fodde R, Smits R (2001) Disease model: familial adenomatous polyposis Trends Mol Med 7: 369-373.

15. Fishel R, Lescoe MK, Rao MR, Copeland NG, Jenkins NA et al. (1993) The human mutator gene homolog $\mathrm{MSH} 2$ and its association with hereditary nonpolyposis colon cancer. Cell 75: 1027-1038.

16. Lynch HT, de la Chapelle A (2003) Hereditary colorectal cancer. N Engl J Med 348: 919-932.

17. Lynch HT, Smyrk T (1996) Hereditary nonpolyposis colorectal cancer (Lynch syndrome). An updated review. Cancer 78: 1149-1167.

18. Tseng W, Leong X, Engleman E (2007) Orthotopic mouse model of colorecta cancer. J Vis Exp 484. 